(2) Open Access Full Text Article

\title{
Retroperitoneal robot-assisted laparoscopic upper pole heminephrectomy in adult patients with duplex kidneys
}

This article was published in the following Dove Press journal:

Therapeutics and Clinical Risk Management

\section{Jie Qin \\ Ping Wang \\ Taile Jing \\ Debo Kong \\ Sunyi Ye \\ Dan Xia \\ Shuo Wang}

Department of Urology, First Affiliated Hospital, Zhejiang University School of Medicine, Hangzhou, Zhejiang Province, People's Republic of China
Correspondence: Dan Xia

Department of Urology, First Affiliated Hospital, Zhejiang University School of Medicine, 79 Qingchun Rd, Hangzhou

310003, Zhejiang Province, People's

Republic of China

$\mathrm{Tel}+8657187236833$

Fax +865718707 2577

Email xiadan@zju.edu.cn
Background: To report our experience with retroperitoneal robot-assisted laparoscopic upper pole heminephrectomy in adult patients with duplex kidneys.

Methods: We retrospectively reviewed the medical records of 7 patients who underwent retroperitoneal robot-assisted laparoscopic upper pole heminephrectomy at our institution between September 2014 and July 2017. Of the robot-assisted laparoscopic procedures, 5 were on the left and 2 on the right side.

Results: All patients underwent robot-assisted laparoscopic surgery successfully in a totally retroperitoneal manner without conversion to open surgery. The mean operative time was 175 mins (range 140-270). The mean estimated blood loss was $84 \mathrm{~mL}$ (range 20-200). The mean postoperative hospital stay was 7 days (range 5-9). No major intraoperative and postoperative complications occurred. All patients had a resolution of their presenting symptoms after surgery at a mean follow-up of 24 months (range 14-38).

Conclusion: Our initial clinical experience suggests that robot-assisted laparoscopic upper pole heminephrectomy using a retroperitoneal approach for a duplex kidney appears to be safe with acceptable perioperative outcomes.

Keywords: robotic, laparoscopy, duplex kidney, heminephrectomy

\section{Background}

A heminephrectomy of the affected moiety is the standard surgical treatment in symptomatic patients with duplex kidneys if necessary. Jordan ${ }^{1}$ reported the first standard laparoscopic upper pole heminephrectomy in a child with a duplex kidney in 1993. The majority of studies on the laparoscopic upper pole heminephrectomy were in childhood. $^{2-4}$ To our knowledge, Patel et $\mathrm{al}^{5}$ described the first robotassisted heminephrectomy in adults in 2010. Typically robot-assisted laparoscopic heminephrectomy in adults has been performed via a transperitoneal approach. We describe a novel robot-assisted laparoscopic upper pole heminephrectomy technique using a retroperitoneal approach that to our knowledge has not been previously described in the literature. ${ }^{6}$

\section{Methods}

\section{Patients}

From September 2014 to July 2017, retroperitoneal robot-assisted laparoscopic upper pole heminephrectomy was performed in 2 men and 5 women who were 20-45 years 
old (median age 24 years). The left kidney was affected in 3 patients, the right kidney was affected in 1 patient and both kidneys were affected in 3. Of the robot-assisted laparoscopic procedures, 5 patients were on the left and 2 patients on the right side. The primary indications for surgery were flank pain, recurrent urinary tract infections (UTIs), abdominal pain and recurrent UTIs. In our study, the major clinical symptoms included flank pain in 3 patients and recurrent urinary tract infection in 4 . The demographics and symptoms of all 7 patients are listed in Table 1.

All patients were evaluated preoperatively using ultrasonography, computed tomography urography or magnetic resonance urography. All 7 patients had a complete, duplicated renal collecting system. The parenchyma of the upper pole was thin and demonstrated minimal function in all patients. Voiding cystourethrography confirmed no vesicoureteral reflux was present in these patients. Urine analysis and culture and renal function tests were all performed. Follow-up studies were performed with ultrasonography and IVU at 3 months postoperatively and annually thereafter. The Medical Research Ethics Board of the First Affiliated Hospital, Zhejiang University School of Medicine, approved this

Table I Demographics and symptoms of 7 patients

\begin{tabular}{|l|l|l|l|l|}
\hline Patient & Age & Sex & Side & Symptoms \\
\hline I & 20 & M & R & Flank pain \\
2 & 45 & F & L & Urinary tract infection \\
3 & 26 & F & L & Flank pain \\
4 & 29 & F & L & Urinary tract infection \\
5 & 24 & F & L & Urinary tract infection \\
6 & 2 I & F & L & Urinary tract infection \\
7 & 22 & M & R & Flank pain \\
\hline
\end{tabular}

retrospective study and waived patient consent for review of medical images and records, as all data were anonymized. This study was conducted in accordance with the Declaration of Helsinki.

\section{Surgical technique}

Under general anesthesia, the patient was placed in the flank position with the ipsilateral side up and secured to the operating table. A 4-port, finger- and balloon-dissecting extraperitoneal laparoscopic approach was used as previously described. ${ }^{7,8}$ A 2-cm transverse skin incision was first made over the iliac crest at the midaxillary line. After blunt dissection with the index finger, a balloon dilator was inserted into the retroperitoneal working space and dilated using air. Under guidance with the finger, three trocars were placed as follows: an $8 \mathrm{~mm}$ robotic trocar at the subcostal posterior axillary line, an 8-mm robotic trocar at the subcostal anterior axillary line and a 12-mm trocar at the level of iliac crest in the anterior axillary line. A fourth trocar was inserted into the 2-cm incision as a robotic camera port (Figure 1A and B). After pneumoperitoneum was created with $\mathrm{CO}_{2}$ gas, a da Vinci (Intuitive Surgical, CA) robot was then docked.

Initially, the retroperitoneal fat was freed, and the Gerota fascia was incised longitudinally. After complete dissection of the dilated upper pole ureter (Figure 2A), the ureters are followed cephalically toward the level of the renal hilum to identify the hilar anatomy. The upper pole vasculature was dissected free (Figure 2B), ligated using Hem-o-lok clips and divided. By cranial traction of the upper pole's ureter, the renal upper pole moiety is then divided using a monopolar scissor along the demarcation line between the upper and lower pole (Figure 2C). Renorrhaphy was performed using with 2-0 V-Loc
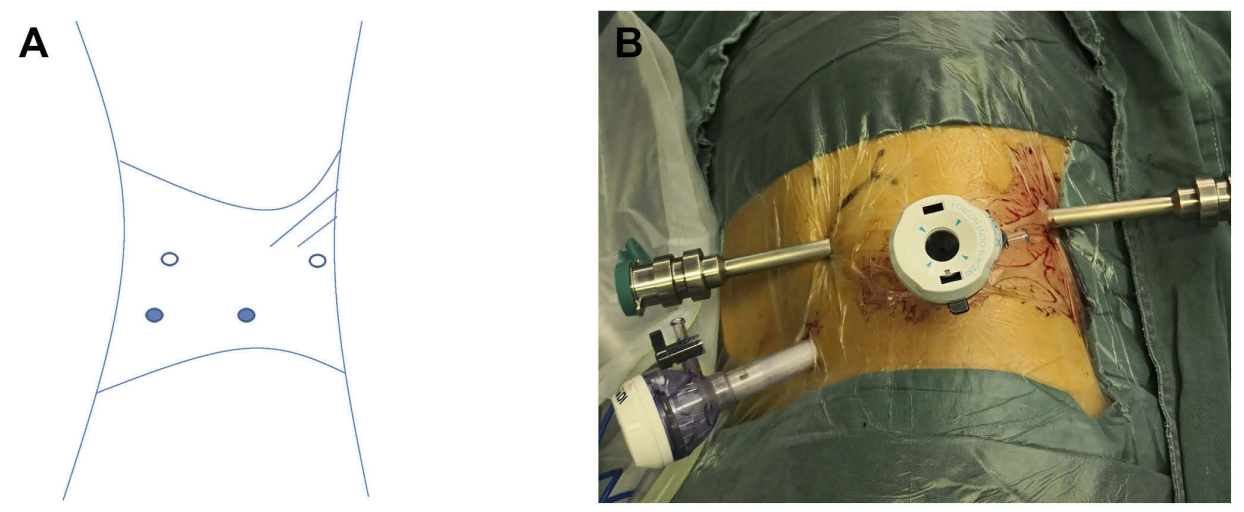

Figure I Port placement for retroperitoneal robot-assisted laparoscopic upper pole heminephrectomy. (A) Trocar locations. Two I2-mm ports were placed and used as a camera port and an assistant port. Two 8-mm ports were placed for the remaining robotic arms. Open circles represent 8-mm ports, and closed circles I2-mm ports. (B) Patient positioning for left dissection. 

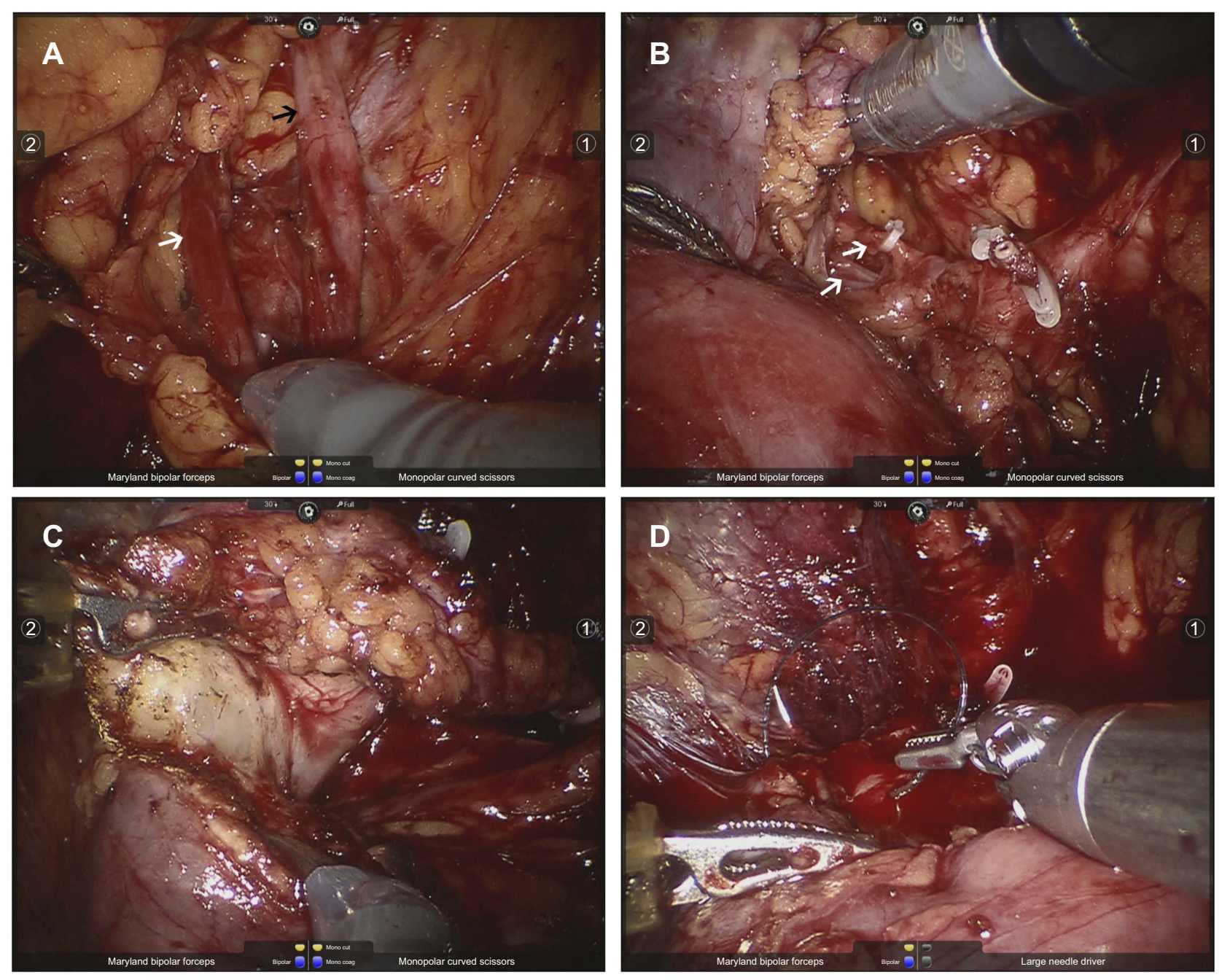

Figure 2 Surgical video screenshots of the retroperitoneal robot-assisted laparoscopic upper pole heminephrectomy. (A) The dilated upper (black arrow) and lower (white arrow) ureters were clearly identified. (B) The upper pole vessels (white arrow) were dissected free, ligated using Hem-o-lok clips. (C) By cranial traction of the proximal ureteral stump, dissection proceeded along the demarcation line between the upper and lower pole. (D) Renorrhaphy was performed.

(Figure 2D). The distal upper pole ureter was mobilized at the level of the iliac vessels, ligated with Hem-o-lok clips and divided. At the end of the procedure, all tissue specimens were retrieved with an endoscopic bag and extracted from the retroperitoneal cavity through the $12-\mathrm{mm}$ assistant port.

\section{Results}

All procedures were completed successfully in a totally retroperitoneal manner without conversion to open surgery. The mean operative time was (skin to skin) 175 mins (range 140-270). Dissection of the upper pole was technically difficult in one case with recurrent UTI as expected. The mean estimated blood loss was $84 \mathrm{~mL}$ (range 20-200), and no blood transfusion was required. Oral intake was resumed between the first and second postoperative day. The drain was removed about 3 days (range 2-4) after surgery. The mean postoperative hospital stay was 7 days (range 5-9). No major intraoperative and postoperative complications occurred. With the mean follow-up of 24 months (range 14-38), all patients had a resolution of their presenting symptoms after surgery. Postoperative imaging including IVU and renal ultrasonography in all patients demonstrated functioning lower pole moiety with an intact collecting system.

\section{Discussion}

Duplex kidney is a relatively common congenital anomaly of the genitourinary tract. The reported incidence is $0.8 \%$ and affects females more than males. ${ }^{9}$ Patients usually present in childhood; however, usually goes asymptomatic until adulthood. In adults, flank pain or recurrent UTI are 
the most commonly associated symptoms. The recommended surgical treatment for this condition is heminephrectomy. One of the limiting factors associated with open surgery is the morbidity and the cosmesis and pain of the open flank incision, especially for adult patients. The first laparoscopic heminephrectomy in a child with a duplex kidney was described by Jordan ${ }^{1}$ in 1993 . Since then, there have been few published reports of laparoscopic upper pole heminephrectomies by both the retroperitoneal and transperitoneal approaches, with the majority being in the pediatric population., ${ }^{2,4}$

Compared to the conventional open surgery, laparoscopic upper pole heminephrectomy has reduced the morbidity of the procedure. Despite its advantages, the laparoscopic procedures present a challenge to the ergonomics of surgery. The main problem of laparoscopic upper pole heminephrectomy is its long and steep learning curve. The da Vinci Surgical System allows for better visualization (the three-dimensional visualization) and increased freedom of movement. However, the cost of robotic surgery is significant, with mean hospital billing amount in our series of $\$ 11,500$.

In the adult literature, the available robotic treatment for duplicated collecting system to date is rare. To our knowledge, the first robot-assisted heminephrectomy in adults was described by Patel et $\mathrm{al}^{5}$ in 2010 . They presented four patients with congenital renal abnormalities diagnosed in adulthood and managed using a robotic procedure. One had a duplex kidney and underwent robotassisted heminephrectomy. The largest available robotic series to date in the adult population was published by Akca et $\mathrm{al}^{10}$ and included 5 patients. The median operative time was 240 mins (range 192-382), estimated blood loss was $150 \mathrm{~mL}$ (range 50-400) and the median length of stay was 3 (2-5) days. Although limited by its retrospective nature, this report demonstrated that robotic procedure can be a viable minimally invasive treatment option for patients with duplex kidney. Mason et $\mathrm{al}^{11}$ also reported their experience with four adult patients. They reported the median operative time was 310 mins (range 240-400), estimated blood loss was $310 \mathrm{~mL}$ (range 80-1200) and the median length of stay was 2.3 (2-3) days. Mean follow-up was 13 (range 9-19) months.

Laparoscopic upper pole heminephrectomy can be performed using a transperitoneal or retroperitoneal approach. ${ }^{12-14}$ The extraperitoneal approach is relatively unique in urological laparoscopic surgery. Most laparoscopic upper pole heminephrectomy is performed via a transperitoneal approach, because it has the advantage of a larger working space. Moreover, complete ureterectomy can be performed when needed. However, it typically involves significant bowel mobilization and manipulation. Several extraperitoneal laparoscopic upper pole heminephrectomy cases have been reported. ${ }^{15}$ Many scholars believed that the retroperitoneal laparoscopic procedure has superior bowel function recovery than the transperitoneal route.

Typically robot-assisted heminephrectomy in adults has been performed via a transperitoneal approach. We describe a novel robotic upper pole heminephrectomy technique using a retroperitoneal approach. Similarly, our perioperative outcomes demonstrate the effectiveness and low morbidity of the retroperitoneal robot-assisted laparoscopic upper pole heminephrectomy in the adult population. However, retroperitoneal robot-assisted upper pole heminephrectomy for a duplex kidney in pediatric patients has been reported. To our knowledge, we describe the first published cases of a robot-assisted laparoscopic heminephrectomy technique using a retroperitoneal approach for adult patients, and our series of 7 adult patients is the largest to date.

\section{Conclusion}

In conclusion, retroperitoneal robot-assisted laparoscopic upper pole heminephrectomy for a duplex kidney in adult patients appears to be safe with acceptable perioperative outcomes. However, our present study is limited by the nature of being a retrospective review and the small sample size. Further, long-term follow-up in a greater number of patients are needed for further studies to determine the role of different approaches to robot-assisted laparoscopic upper pole heminephrectomy.

\section{Acknowledgment}

This study was supported by grants from the Natural Science Foundation of Zhejiang Province (LY18H050001, LQ16H030002).

\section{Disclosure}

The authors report no conflicts of interest in this work.

\section{References}

1. Jordan GH, Winslow BH. Laparoendoscopic upper pole partial nephrectomy with ureterectomy. $J$ Urol. 1993;150(3):940-943. doi:10.1016/S0022-5347(17)35656-2 
2. Esposito C, Miyano G, Caione P, et al. Retroperitoneoscopic heminephrectomy in duplex kidney in infants and children: results of a multicentric survey. J Laparoendosc Adv Surg Tech A. 2015;25 (10):864-869. doi:10.1089/lap.2014.0654

3. Herz D, Smith J, McLeod D, Schober M, Preece J, Merguerian P. Robotassisted laparoscopic management of duplex renal anomaly: comparison of surgical outcomes to traditional pure laparoscopic and open surgery. J Pediatr Urol. 2016;12(1):44.e1-44.e7. doi:10.1016/j.jpurol.2015.04.046

4. Esposito C, Escolino M, Miyano G, et al. A comparison between laparoscopic and retroperitoneoscopic approach for partial nephrectomy in children with duplex kidney: a multicentric survey. World J Urol. 2016;34(7):939-948. doi:10.1007/s00345-015-1728-8

5. Patel MN, Kaul SA, Bhandari A, et al. Robot-assisted management of congenital renal abnormalities in adult patients. J Endourol. 2010;24 (4):567-570. doi:10.1089/end.2009.0313

6. Clement K, Hendry J, Keeley FX, Aboumarzouk OM. Heminephrectomy in adults: a systematic review with cumulative analysis. Urol Int. 2018;101 (1):1-6. doi:10.1159/000485466

7. Wang P, Xia D, Ma Q, Wang S. Retroperitoneal laparoscopic management of ureteropelvic junction obstruction in patients with horseshoe kidney. Urology. 2014;84(6):1351-1354. doi:10.1016/j.urology.2014.07.029

8. Qin J, Wang P, Jing T, Kong D, Xia D, Wang S. Extraperitoneal robot-assisted laparoscopic retroperitoneal lymph node dissection for early-stage testicular nonseminomatous germ cell tumors: a case report and literature review. Medicine. 2017;96(49):e8938. doi:10.1097/ MD.0000000000008938
9. Didier RA, Chow JS, Kwatra NS, Retik AB, Lebowitz RL. The duplicated collecting system of the urinary tract: embryology, imaging appearances and clinical considerations. Pediatr Radiol. 2017;47(11):1526-1538. doi:10.1007/s00247-017-3904-z

10. Akca O, Kaouk JH, Zargar H, et al. Robot assisted heminephrectomy for duplicated renal collecting system: technique and outcomes. Mrcas. 2015;11(2):126-129. doi:10.1002/rcs.1607

11. Mason MD, Peters CA, Schenkman NS. Robot-assisted upper pole nephrectomy in adult patients with duplicated renal collecting systems. $J$ Endourol. 2012;26(7):838-842. doi:10.1089/ end.2011.0377

12. Li HZ, Ma X, Zhang J, et al. Retroperitoneal laparoscopic upper-pole nephroureterectomy for duplex kidney anomalies in adult patients. Urology. 2011;77(5):1122-1125. doi:10.1016/j.urology.2010.06.073

13. Gao Z, Wu J, Lin C, Men C. Transperitoneal laparoscopic heminephrectomy in duplex kidney: our initial experience. Urology. 2011;77 (1):231-236. doi:10.1016/j.urology.2010.06.073

14. Abouassaly R, Gill IS, Kaouk JH. Laparoscopic upper pole partial nephrectomy for duplicated renal collecting systems in adult patients Urology. 2007;69(6):1202-1205. doi:10.1016/j.urology.2007.03.011

15. Chen Z, Tang ZY, Fan BY, et al. Retroperitoneoscopic upper pole nephroureterectomy in duplex kidney: focus on the role of dilated upper pole ureter. Urol J. 2014;10(4):1046-1053.
Therapeutics and Clinical Risk Management

\section{Publish your work in this journal}

Therapeutics and Clinical Risk Management is an international, peerreviewed journal of clinical therapeutics and risk management, focusing on concise rapid reporting of clinical studies in all therapeutic areas, outcomes, safety, and programs for the effective, safe, and sustained use of medicines. This journal is indexed on PubMed Central, CAS,

\section{Dovepress}

EMBase, Scopus and the Elsevier Bibliographic databases. The manuscript management system is completely online and includes a very quick and fair peer-review system, which is all easy to use. Visit http://www.dovepress.com/testimonials.php to read real quotes from published authors. 